\title{
Application of Luenberger Shortage Function on the Zagreb Stock Exchange: Analysis of Efficient Market Portfolios
}

\author{
Denis Dolinar ${ }^{1}$, Tihana Škrinjarić1 ${ }^{1,}$ and Davor Zoričicić ${ }^{1}$ \\ ${ }^{1}$ Faculty of Economics and Business, University of Zagreb, Trg J. F. Kennedyja 6, 10000 Zagreb, Croatia \\ E-mail: <\{ddolinar, tskrinjaric,dzoricic\}@efzg.hr>
}

\begin{abstract}
In order to apply the CAPM in practice, the estimation of the market portfolio presents one of the biggest challenges. Even more so after the research in early 1990s pointed out the mean-variance inefficiency of stock market capitalization-weighted indices. In the paper we perform an in-sample optimisation for the CROBEX index constituents to test its efficiency ex post. Selected CROBEX index revisions from the period March 2005 - September 2017 are analysed. We find the index to be inefficient which confirms the results of earlier studies. However, since mean-variance optimisation often yields extreme portfolio weights, thus reducing the effective number of stocks in the portfolio, the focus of this research is on testing if improvement in efficiency over the CROBEX index is possible, with respect to the portfolio deconcentration level. The Luenberger's shortage function is added to the optimisation algorithm in order to increase the number of efficient portfolios analysed.
\end{abstract}

Keywords: Croatian stock market, efficient frontier, efficient market portfolio, shortage function

Received: April 23, 2018; accepted: September 13, 2018; available online: December 13, 2018

DOI: 10.17535 /crorr.2018.0014

\section{Introduction}

Since the work of Haugen and Baker [15] and Grinold [10], who first pointed out that the stock market capitalization-weighted indices provided an inefficient risk-reward ratio, research efforts focused on strategies which could improve asset allocation efficiency. Even though Zoričić et al. [23], following the work of Amenc et al. [1] for the developed markets, found the CROBEX index to be inefficient, an attempt to implement an efficient strategy in the Croatian equity market, done by Dolinar et al. [8], failed dismally. Further research by Habibović et al. [12] confirmed some of the earlier findings, but also stressed the advantages of using the broad-based CROBEX over the CROBEX10 index, and that instead of measuring the Euclidean distance of CROBEX from the closest portfolio on its efficient frontier, multiple efficient portfolios should be analysed, taking into account the indifference level of transaction costs.

\footnotetext{
${ }^{*}$ Corresponding author.

http://www.hdoi.hr/crorr-journal

@2018 Croatian Operational Research Society
} 
In this research we attempt to perform just such an analysis by following Briec et al. [6], who used the Luenberger's distance function to gauge the performance of portfolios in the mean-variance context. Since this approach allows to gauge performance in any direction, it generalises existing approaches of measuring inefficiency, which makes it a perfect tool for a comprehensive assessment of the CROBEX index's in-sample inefficiency. Namely, the above mentioned research results suggest that regardless of the CROBEX' inefficiency, outperforming the cap-weighted index from the out-of-sample perspective can be hard to achieve in the illiquid and undeveloped market. Therefore, pursuing the efficient allocation strategies, presented for example in Amenc et al. [4], and testing them out-of-sample in a such specific environment can prove to be futile. Instead, in this paper we turn to the proposed methodology to provide a thorough in-sample analysis as a prerequisite for such future efforts. We analyse 2 most common efficient strategies, the Maximum Sharpe Ratio (MSR) and Global Minimum Variance (GMV) portfolios, but also 4 additional efficient portfolios (presenting a novelty since they are not commonly mentioned as investment strategies), which we define by the Luenberger's distance function. The main goal of the research is, therefore, to determine the efficient portfolios' out-ofsample estimation potential based on such in-sample efficiency analysis.

As pointed out by some authors, for instance in Amenc et al. [4] and Amenc et al. [5], the MSR portfolio has to perform the best in-sample, being the optimal portfolio according to the Modern Portfolio Theory as pointed out by Tobin [22]. However, whether it performs well enough to account for the transaction costs even on the in-sample basis hasn't been looked into on the Croatian stock market. Also, from the out-of-sample perspective its estimation risk is the highest since it requires the estimation of 3 parameters. On the other hand, the GMV portfolio requires estimation of one less parameter, but may fail to perform well even in-sample due to low returns associated with low volatility, which is even more pronounced in the Croatian market conditions. Due to estimation risk, poor in-sample performance implies worse out-ofsample performance, therefore we provide the analysis of additional efficient portfolios in order to provide a complete picture of the research problem at hand, based on which the efforts to estimate efficient portfolios out-of-sample could be evaluated.

Additionally, a drop in the performance of selected efficient portfolios as higher portfolio deconcentration is imposed is taken into account in the analysis, since CROBEX is relatively well deconcentrated (primarily due to the low number of constituents ${ }^{1}$ and due to the imposed restriction on maximum constituent weight of 10\%) compared to its developed markets' counterparts. Therefore, unlike for the developed markets, naïve strategies such as equalweighing do not perform well, let alone outperform the CROBEX. On the other hand, optimisation-based strategies may lead to heavy concentration in a few stocks, which leads to good performance in-sample but is accompanied with a lot of estimation risk from an out-ofsample perspective. Hence, the analysis carried out in this paper should help future research efforts, seeking to provide efficient benchmarks in undeveloped and illiquid financial markets, pursue viable strategies. The structure of the paper is as follows. The second section describes

${ }^{1}$ In the observed period March 2005 - September 2017 the average number of constituents stood at approximately 25 . 
the methodology used in the empirical part of the paper, namely the third and fourth sections. The final, fifth section concludes the paper with recommendations for future research.

\section{Methodology}

The cornerstone of the applied methodology is the Markowitz [19, 20] model:

$$
\begin{array}{cl}
\max _{\boldsymbol{w}} & \boldsymbol{w}^{\prime} \boldsymbol{E}(\boldsymbol{R}) \\
\text { s.t. } & \boldsymbol{w}^{\prime} \boldsymbol{\Sigma} \boldsymbol{w} \leq c \\
& \boldsymbol{e} \boldsymbol{w}=1 \\
& \boldsymbol{w} \geq \mathbf{0}
\end{array}
$$

where $\boldsymbol{w}$ is $(N \cdot 1)$ the vector of stock shares in the portfolio, $\boldsymbol{E}(\boldsymbol{R})$ is $(1 \cdot N)$ the vector of expected returns, $\boldsymbol{\Sigma}(N \cdot N)$ the positive semi-definite variance-covariance matrix, $\boldsymbol{e}(N \cdot 1)$ the unit vector, $c$ is the risk level, which the investor can change due to his preferences, and $\mathbf{0}$ is $(N \cdot 1)$ the vector of null values. By changing the value of $c$ the investor can derive the efficient frontier in order to select the optimal portfolio from that frontier. Based upon the assumptions behind the Markowitz model and expanding the distance functions from production economics, Briec et al. [6] developed the methodology to gauge performance of portfolios in the meanvariance context. They integrated an efficiency measure in that model in order to evaluate inefficiency of any portfolio which is not on the efficient frontier resulting from that model. In that way, any inefficient portfolio can be gauged with respect to the efficient frontier. Moreover, sources of inefficiencies can be found, ranging from allocative and portfolio to overall inefficiency. This measure is a variation of a shortage function from production theory, introduced by Luenberger [18]. Luenberger's function is a distance function which simultaneously looks for expansions in outputs and reductions in inputs. Briec et al. [6:5] added additional properties needed for the set of feasible portfolios in order to use this function in the portfolio context, so optimisation can be carried out over a free disposal hull. All properties and proofs are given in Briec et al. [6:7-8]. Here, the main model is summarized. We observe portfolio $j$ with properties $\left(\sigma_{j}^{2}, E(R)_{j}\right)=\left(\boldsymbol{w}_{j}^{\prime} \boldsymbol{\Sigma} \boldsymbol{w}_{j}, \boldsymbol{w}_{j}^{\prime} \boldsymbol{E}(\boldsymbol{R})_{j}\right)$ in the variance-return space, $j \in\{1,2, \ldots, J\}$, for which performance needs to be gauged, i.e. portfolio structure $\boldsymbol{w}_{j}$, variance and return are known. The shortage function, named efficiency improvement possibility (EIP) function of this portfolio, is defined as:

$$
\begin{array}{ll}
\max _{\boldsymbol{w}} & \delta \\
\text { s.t. } & \boldsymbol{w}_{j}^{\prime} \boldsymbol{\Sigma} \boldsymbol{w}_{j}-\delta g_{\Sigma} \geq \boldsymbol{w}^{\prime} \boldsymbol{\Sigma} \boldsymbol{w} \\
& \boldsymbol{w}_{j}^{\prime} \boldsymbol{E}_{j}(\boldsymbol{R})+\delta g_{E(R)} \leq \boldsymbol{w}^{\prime} \boldsymbol{E}(\boldsymbol{R}) \\
& \boldsymbol{e} \boldsymbol{w}=1 \\
& \boldsymbol{w} \geq \mathbf{0}
\end{array}
$$


where $\left(g_{\Sigma}, g_{E(R)}\right)$ is the directional vector which defines in which direction the inefficient portfolio should shift in order to arrive on the efficient frontier. Optimal value $\delta^{*}$, which results from optimising (2), is the improvement needed regarding both an increase in portfolio return and reduction in portfolio risk. Selection of the directional vector can affect the rankings, and depends upon the observed problem (see Kerstens et al. [17]). In this study we chose (1,1) in order to achieve the fastest growth of return and fastest reduction of risk. Unfortunately, the literature does not recommend specific direction, as [17] state that at this point, every direction the authors analysed has its own merits and drawbacks. E.g. the PD (position dependent) scheme has advantage over other schemes in terms of proportional interpretation. However, its drawback is ranking the portfolios with lower variance and returns as more efficient compared to portfolios with higher variance and returns. Since the purpose of this paper is to analyse improvements of efficiency of the market portfolio (the CROBEX index), a parsimonious approach is made to achieve the efficiency in the fastest way. Other model specifications are also analysed. Firstly, only the return is maximized in (2), with the risk set to value of the market portfolio. Next, the risk was minimized with the return set to the value of market portfolio return. Third variation of the model is analysed with equal value of $\delta$ in both risk and return constrains, i.e. the model (2) is analysed. Final variation of the model is analysed with individual values $\delta_{\text {return }}$ and $\delta_{\text {risk }}$ in their respective constraints.

Since the CROBEX return pattern (as presented in the following section in the Figure 1) exhibited regime switching behaviour in the analysed period, a Markov regime switching (MRS) model was utilized in order to classify CROBEX revision periods as belonging to a high or low volatility regime. It is assumed that the observed stock market return follows a MRS of order one, $r_{t} \sim N\left(\mu, \sigma_{t, k}^{2}\right)$, where $k$ denotes the number of regimes, $k \in\{1,2\}$. Volatility is modelled as a GARCH $(1,1)$ process in each regime, by using the maximum likelihood method and the Gray [9] algorithm of optimisation. Justification of two regimes on the Zagreb Stock Exchange (ZSE) is given in Škrinjarić and Šego [21] and details on the model and estimation procedure are given in Hamilton [13] and [14], Gray [9] or Haas et al. [11].

\section{Data and preliminary calculations}

This research is focused on the CROBEX index, representing a broad-based index (and also the oldest index) in the Croatian capital market. The broad-based index is chosen because the greater the number of constituents in the index, the greater the opportunity for diversification. This is especially important in a small market since (as shown in Table 1) the number of constituents never exceeded 30 in the analysed period, and today stands at 25 . The period ranging from March 2005 till September 2017 was selected for the analysis as Croatian economy experienced expansion, recession and recovery during the specified time frame. The specified months for the beginning and the end of the observation period correspond to regular revisions of the index, which occur twice a year (in March and September). Since the objective of this paper is to analyse the efficiency of the CROBEX index by deriving its efficient frontier based on the return data of its constituents, CROBEX index revisions were treated as individual data samples. Therefore, only those revisions for which the CROBEX structure remained unchanged 
over the course of the revision period were considered in order to maximize the number of observations in each revision (sample). Because of this, in the analysed period, 25 regular revisions were trimmed to 19 and then further to 14 as only the revisions in which expansion, recession and recovery of the economy was pronounced the most were kept as selected samples from the overall data. The selected revisions are presented in the Table 1.

\begin{tabular}{|c|c|c|}
\hline Revision (sample) & Weeks of the year & Number of stocks \\
\hline $2005 \quad \mathrm{R} 1$ & $12-37$ & 17 \\
\hline 2005 R2 & $38(2005)-11(2006)$ & 21 \\
\hline 2006 R1 & $12-37$ & 23 \\
\hline $2006 \_$R2 & $38(2006)-11(2007)$ & 25 \\
\hline $2008 \quad \mathrm{R} 1$ & $13-38$ & 30 \\
\hline $2008 \quad \mathrm{R} 2$ & $39(2008)-12(2009)$ & 28 \\
\hline $2009 \_\mathrm{R} 1$ & $13-38$ & 24 \\
\hline $2009 \_\mathrm{R} 2$ & $39(2009)-11(2010)$ & 24 \\
\hline $2011 \quad \mathrm{R} 1$ & $12-37$ & 25 \\
\hline $2012 \quad \mathrm{R} 2$ & $39(2012)-11(2013)$ & 25 \\
\hline $2013 \quad \mathrm{R} 1$ & $12-38$ & 25 \\
\hline $2014 \_\mathrm{R} 1$ & $13-38$ & 25 \\
\hline $2015 \quad \mathrm{R} 1$ & $13-38$ & 25 \\
\hline $2016 \quad \mathrm{R} 1$ & $12-37$ & 23 \\
\hline
\end{tabular}

Table 1: Selected CROBEX index revision periods for the analysis (source: Zagreb Stock Exchange)

Weekly excess ${ }^{2}$ total returns (in excess of the risk free rate ${ }^{3}$ ) of CROBEX index constituents were used in the analysis. Weekly data on stock prices and CROBEX structure were downloaded from the ZSE. Data on dividends were downloaded from the Central Depository \& Clearing Company Inc., and data on Treasury bill yield from the Croatian National Bank and Ministry of Finance. Based on the collected data for each revision, the risk and return of CROBEX index were calculated as for any other portfolio in the analysis. Therefore, the risk-return characteristics of the CROBEX index for the analysed period correspond to the CROBEX total return index.

\footnotetext{
${ }^{2}$ Excess returns can be viewed as an indicator of portfolio profitability relative to the return on the risk free asset. Also, it can be noted that by using the excess return the collected data represents the numerator of the Sharpe ratio, which is explained and used to measure portfolio performance later in the text.

${ }^{3}$ The returns on Treasury bills are used as a standard proxy for the risk free rate due to the fact that they represent an investment with the lowest risk in a market as a short term government security.
} 


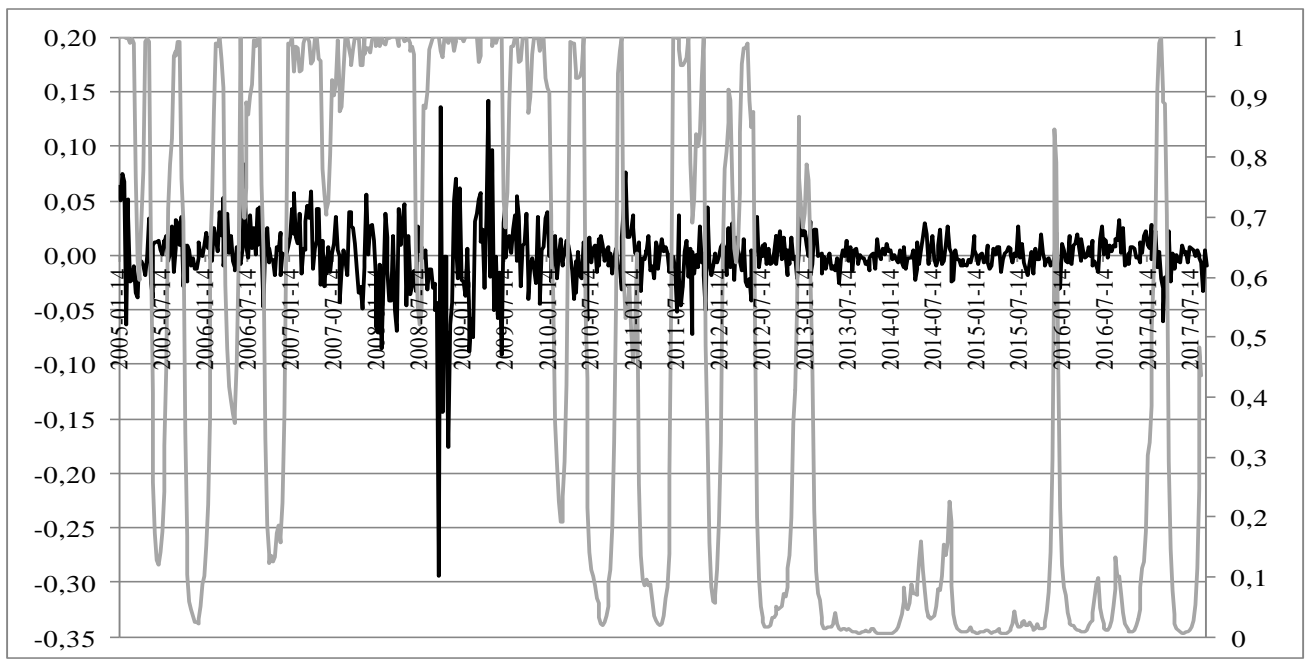

Figure 1: CROBEX return (black line, left axis) and smoothed probability of a high risk regime (grey line, right axis) (source: authors)

Preliminary analysis included observing properties of risk and return in order to detect regimes of high and low market volatility. In that way, possible patterns in each regime can be seen more easily. Stock market return was filtered in the first step as an $\operatorname{ARMA}(1,1)$ process $^{4}$. In the second step, a Markov regime switching model was estimated, with assumption of two regimes, which correspond to high and low volatility ${ }^{5}$, with the assumption of GARCH $(1,1)$ process in each regime. The results of the analysis presented in the Figure 1 suggest that since the second half of 2012 the low volatility regime dominates the high volatility regime. Therefore, out of total of 14 CROBEX index revision periods, 9 were classified as belonging to the high volatility regime (2005_R1 - 2011_R1) and 5 as belonging to the low volatility regime (2012_R2-2016_R1).

\section{Results of the analysis}

By applying the previously described methods to the collected data we estimated the efficient portfolios for each of the analysed CROBEX index revision periods. We began by using the original Markowitz model and estimating the Global Minimum Variance (GMV) and Maximum

\footnotetext{
${ }^{4}$ Box-Jenkins approach of $\operatorname{ARMA}(p, q)$ modelling was applied, where $\operatorname{ARMA}(1,1)$ model was best based upon model diagnostics. Detailed results are available upon request. In order to complete the regime switching modelling, ARMA estimation was carried out on the return series in order to decrease the number of parameters which needed to be estimated via MRS methodology. Residuals were then extracted (filtering the ARMA model) and as they were free of ARMA effects they were used in the next step of regime switching modelling. This is the usual approach when several steps in modelling have to be carried out, for details see Chen [7].

${ }^{5}$ Again, detailed results are available upon request.
} 
Sharpe Ratio (MSR) portfolios ${ }^{6}$. By adding the EIP function (2) to the analysis, the model was optimised for (i) only the portfolio return, (ii) only the portfolio risk, (iii) both risk and return and (iv) both risk and return with individual $\delta_{\text {return }}$ and $\delta_{\text {risk. }}$. Thus, we optimised portfolios in relation to the CROBEX index estimating: (i) Risk Maximization (RM), (ii) Risk Reduction (RR), (iii) GAMA and (iv) GAMAs efficient portfolios. Since the short selling is not allowed on the ZSE only the $\mathbf{w} \geq \mathbf{0}$ constraints were imposed.

However, since the optimisation procedure is known to lead to extreme positions in investments comprising the portfolio, causing high concentration in a few stocks, further constraints are often imposed to portfolio weights (especially with the out-of-sample estimation in mind) ${ }^{7}$. Therefore, following the work of Amenc et al. [5], we introduced the following constraints:

$$
w_{i, \min }=\frac{1}{\lambda N} \leq w_{i} \leq \frac{\lambda}{N}=w_{i, \max } \forall i \in\{1,2, \ldots, N\}
$$

where $\lambda \in \mathbb{N}$ and $N$ is the number of CROBEX, i.e. portfolio constituents in order to insure greater portfolio deconcentration. Under these constraints all of the above-mentioned portfolios were estimated again for each CROBEX revision period with $\lambda$ set to 4 (except for two revisions in 2005 for which $\lambda$ had to be set to 5 since it was not possible to obtain optimal solution for the value set to 4). If $\lambda$ is set to 1 , deconcentration is set to maximum and portfolio becomes equally-weighted. Therefore, the greater the $\lambda$, the less restrictive are the constraints and weaker is the reduction of the efficient frontier. We opted for $\lambda$ set to 4 to analyse the initial impact of the introduction of constraints on the efficient portfolio estimation as it allows for a reasonable degree of freedom in the optimisation process. As reported in Table 1, the number of constituents in the CROBEX index varied from 17 to 30, with an average of slightly above 24 . This implies equally-weighted portfolios with weights in the range from $5.88 \%$ to $3.33 \%$ (for 17 and 30 stocks in portfolio respectively), or slightly above $4 \%$ on average. By introducing the stated values of $\lambda$ the minimum constraint was set, on average, to slightly over $1 \%$ and the maximum constraint to $17.5 \%$.

\footnotetext{
${ }^{6}$ Maximum Sharpe Ratio (MSR) portfolio is a unique portfolio with the highest Sharpe ratio (SR) as a measure of the risk-adjusted return in the investment universe. In this paper we define the SR as excess expected portfolio return (return above risk-free rate) per one unit of portfolio risk (i.e. standard deviation of return): $S R=\frac{E(R) p}{\sigma_{E(R) p}}$. The MSR portfolio's weights are calculated from (1) by adding the "maximize SR" constraint.

Global Minimum Variance (GMV) portfolio is a unique portfolio with the lowest risk in the investment universe. The GMV portfolio's weights are calculated by solving the following formula: $\boldsymbol{w}^{*}=\arg \underset{\boldsymbol{w}}{\min } \frac{\boldsymbol{\Sigma}^{\mathbf{- 1}} \mathbf{1}}{\mathbf{1}^{-\mathbf{1}} \mathbf{1}}$, where $\boldsymbol{\Sigma}$ is the covariance matrix of expected returns of portfolio constituents meaning that only covariances and variances enter optimisation (but excepted returns do not unlike in the case of MSR portfolio).

${ }^{7}$ From an in-sample perspective one could hold an efficient, yet highly concentrated portfolio, but from an out-of-sample perspective, highly concentrated efficient portfolios are highly sample dependant and exposed to a lot of estimation risk.
} 
As reported later in Table 4, the constraints introduced above still lead to portfolios which are deconcentrated well below the CROBEX index, as can be seen by the effective number of stocks ${ }^{8}$ in a portfolio. So instead of lowering the value of $\lambda$ further to increase the deconcentration of portfolio further, we decided to estimate the chosen efficient portfolios under the constraint that each portfolio level of deconcentration matches the deconcentration of CROBEX index for the given revision period. On average (for 14 revisions), this level of deconcentration stood slightly above $50 \% .{ }^{9}$ We repeated this exercise one more time but this time decided to set the deconcentration level arbitrarily at $75 \%$ for efficient portfolios (close to 25 p.p. higher). The described additional constraints are imposed through formula for the effective number of stocks in a portfolio and are given by inequality (4):

$$
\frac{1}{\sum_{i=1}^{N} w_{i}^{2}} \geq \frac{1}{\sum_{i=1}^{N} w_{i, \text { CROBEX }}^{2}}
$$

In order to remain concise, the results of optimisation procedures in the form of a chart are presented in the Figure 2 for one CROBEX revision period only.

In Figure 2 the position of the efficient portfolios relative to the CROBEX index is depicted for unconstrained and constrained optimisations mentioned above. The "constrained" suffix next to portfolio designations is related to lambda constrained optimisation. "N_Cbex" suffix indicates constrained optimisation leading to the concentration equal to that of the CROBEX index and "75\%" to the deconcentration of $75 \%$ (which will be referred to in more detail a bit later). Apart from the portfolios specified at the beginning of this section, the performance of the equally-weighted (EW) portfolio (presenting a naïve diversification method ${ }^{10}$ ) is also shown.

\footnotetext{
${ }^{8}$ If effective number of stocks is divided by the nominal number of constituents the ratio presents a measure of deconcentration. Therefore, the higher the effective number of stocks in a portfolio, the greater the deconcentration.

9 The reported level of deconcentration corresponds to the effective number of stocks (constituents) of around 12.4 on average.

${ }^{10}$ It should be noted that there is no estimation risk involved as portfolio weights for the EW portfolio are obtained by the $1 / \mathrm{N}$ rule both in- and out-of-sample.
} 


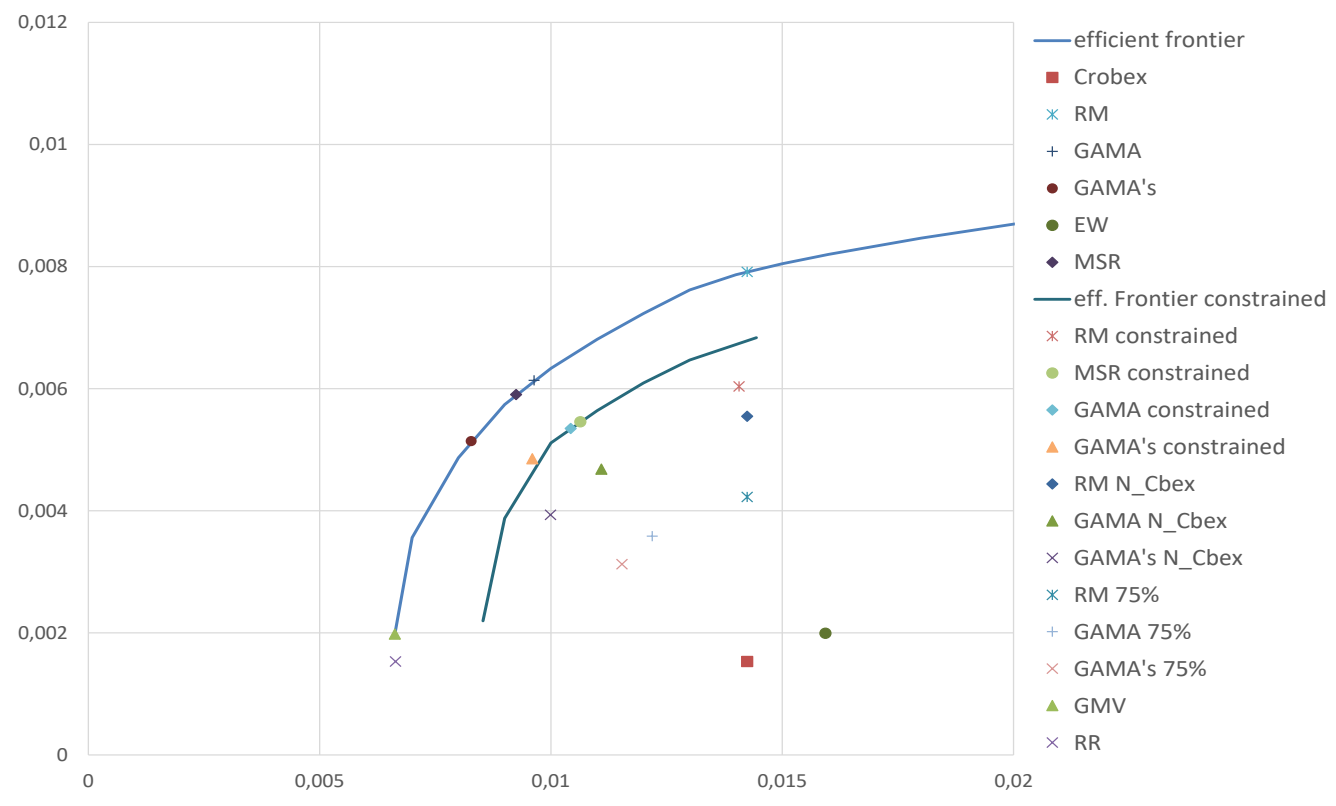

Figure 2: Mean-variance optimization of CROBEX index constituents for the $2005^{\circ} \mathrm{R} 1$ revision of the CROBEX index (source: authors)

For all of the estimated efficient portfolios under each optimisation procedure, the following data are reported: modified Sharpe ratio, effective number of stocks, excess return and excess turnover over CROBEX index and indifference level of transaction costs. The first three measures were calculated on average (for the revision periods analysed) with the third one being calculated as a geometric average and expressed on an annual basis. Excess turnover was calculated in absolute terms but also expressed on an annual basis. Modified Sharpe ratio is used following the work of Israelsen $[16]^{11}$, as it provides a better measure of portfolio performance if negative returns are experienced in the market (which is the case here). Effective number of stocks, as described earlier, indicates the level of deconcentration in the portfolios. Indifference level of transaction costs is calculated, following the work of Amenc et al. [5], by dividing the excess return over CROBEX by excess turnover over CROBEX. Efficient portfolios should provide a better risk-return trade-off, therefore yielding greater Sharpe ratios. If a greater Sharpe ratio is driven by a greater return over CROBEX, the excess return should be sufficient to cover transaction costs associated with the greater (excess) turnover of efficient portfolios. Therefore, the ratio presenting the indifference level of transaction costs provides a measure of transaction

11 Modified Sharpe ratio is calculated by the following formula:
$S R$ modified $=E(R)_{p} / \frac{E(R)_{p}}{\sigma_{E(R)_{p}}^{a b s(l(R) p)}}$, where $E(R)_{p}$ is the portfolio excess return and $\sigma_{E(R) p}$ is the standard deviation of portfolio's excess return. 
costs an investor can incur at which he would be indifferent about investing in an efficient portfolio versus CROBEX.

Table 2 presents the results of unconstrained optimisation (short selling restriction only). The EW portfolio, as expected, does not outperform any of the efficient portfolios but, unlike in the developed markets (see for example Amenc et al. [1]), it does not outperform the CROBEX index (not even in terms of return). Also, a bit surprising is the relatively poor performance of the GMV portfolio with a Sharpe ratio below the CROBEX index caused by, on average, greater reduction in return (smaller return) than in risk relative to CROBEX. Therefore, the RR portfolio (risk reduction only relative to CROBEX) represents a better option when the focus is on risk reduction. However, in this case there cannot be any excess return over CROBEX by definition, meaning that the indifference level of transaction costs stands firmly at 0 . These portfolios are therefore excluded from further analysis.

\begin{tabular}{|l|r|r|r|r|r|r|r|r|}
\hline & CROBEX & \multicolumn{1}{|c|}{ EW } & \multicolumn{1}{c|}{ RR } & GMV & GAMA & GAMAs & RM & MSR \\
\hline Sharpe ratio (modified) & 0.30 & 0.20 & 0.41 & 0.28 & 0.56 & 0.57 & 0.56 & 0.60 \\
\hline $\begin{array}{l}\text { Effective number of } \\
\text { stocks }\end{array}$ & 12 & 24 & 6 & 6 & 5 & 5 & 4 & 5 \\
\hline $\begin{array}{l}\text { Excess return over } \\
\text { CROBEX index }\end{array}$ & $0.00 \%$ & $-11.7 \%$ & $0.00 \%$ & $-18.8 \%$ & $44.49 \%$ & $28.91 \%$ & $76.82 \%$ & $33.17 \%$ \\
\hline $\begin{array}{l}\text { Excess turnover over } \\
\text { CROBEX index }\end{array}$ & $0.0 \%$ & $-2.9 \%$ & $225.4 \%$ & $218.8 \%$ & $243.3 \%$ & $226.2 \%$ & $279.9 \%$ & $247.4 \%$ \\
\hline $\begin{array}{l}\text { Indifference level of } \\
\text { transaction costs }\end{array}$ & - & $N A$ & $0.00 \%$ & $-8.58 \%$ & $18.29 \%$ & $12.78 \%$ & $27.44 \%$ & $13.41 \%$ \\
\hline
\end{tabular}

Table 2: Performance of efficient portfolios compared to the CROBEX index (unconstrained optimisation, full sample - 14 revision periods) (source: authors)

Before introducing constraints on weights, two subsamples (related to two detected regimes) for the same optimisation process are analysed first. Generally, an increase (absolute and also relative to the CROBEX) in the Sharpe ratio of the efficient portfolios can be noticed in the low volatility period. On the other hand, the indifference level of transaction costs seems to be lower in the same period (with the exception of the MSR portfolio). This is shown in the Table 3.

Next, analysis is extended by imposing the constraints on weights (by parameter $\lambda$ as described previously). When unconstrained, the effective number of portfolio constituents ranged from 3 to 6 which, at best, represented only half of the effective number of constituents of the CROBEX index. However, as can be seen in Table 4 below, although the introduction of parameter lambda did improve portfolio deconcentration (effective number of stocks in efficient portfolios rose), it is still well below the CROBEX index. Therefore, further below in the same table we provide results for the efficient portfolios with deconcentration level approximately equal to the CROBEX index and for the deconcentration standing at $75 \%$. For the latter, the results are presented for the full sample and for the two subsamples (the high and low volatility regimes). 


\begin{tabular}{|l|r|r|r|r|r|}
\hline High volatility sample (9 revisions) & CROBEX & GAMA & GAMAs & RM & \multicolumn{1}{c|}{ MSR } \\
\hline Sharpe ratio (modified) & 0.31 & 0.52 & 0.53 & 0.48 & 0.54 \\
\hline Effective number of stocks & 12 & 5 & 4 & 3 & 5 \\
\hline Excess return over CROBEX index & $0.00 \%$ & $61.03 \%$ & $31.93 \%$ & $102.77 \%$ & $31.64 \%$ \\
\hline Excess turnover over CROBEX index & $0.00 \%$ & $267.44 \%$ & $224.35 \%$ & $292.71 \%$ & $248.52 \%$ \\
\hline Indifference level of transaction costs & - & $22.82 \%$ & $14.23 \%$ & $35.11 \%$ & $12.73 \%$ \\
\hline Low volatility sample (5 revisions) & CROBEX & GAMA & GAMA'S & RM & MSR \\
\hline Sharpe ratio (modified) & 0.28 & 0.62 & 0.64 & 0.69 & 0.71 \\
\hline Effective number of stocks & 14 & 6 & 6 & 5 & 5 \\
\hline Excess return over CROBEX index & $0.00 \%$ & $19.54 \%$ & $23.87 \%$ & $38.85 \%$ & $35.78 \%$ \\
\hline Excess turnover over CROBEX index & $0.00 \%$ & $204.71 \%$ & $229.31 \%$ & $259.53 \%$ & $245.63 \%$ \\
\hline Indifference level of transaction costs & - & $9.55 \%$ & $10.41 \%$ & $14.97 \%$ & $14.57 \%$ \\
\hline
\end{tabular}

Table 3: Performance of efficient portfolios compared to the CROBEX index - unconstrained optimisation (source: authors)

Based on the results presented in the Table 4 (but also on the results in Tables 2 and 3) a few observations can be pointed out. First, as deconcentration gets higher the Sharpe ratios of efficient portfolios are becoming lower (the difference is lower in a high volatility period and almost vanishes at portfolio deconcentration level of $75 \%$ ). Second, generally the indifference level of transaction costs is higher in the high volatility subsample (additional data available at request). On the one hand, high returns are accompanied by higher volatility bringing the Sharpe ratios of efficient portfolios closer to CROBEX, but on the other hand high returns are also increasing the indifference level of transaction costs (high excess returns relative to excess turnover). The latter is much less pronounced at the level of deconcentration of $75 \%$, in which case the MSR portfolio dominates over the RM portfolio. This occurs because the RM portfolio only maximizes return relative to the CROBEX index and when, additionally, the $75 \%$ level of the deconcentration constraint is applied, the increase in return over CROBEX is limited. The MSR portfolio on the other hand is able to increase the return much more significantly while meeting the same $75 \%$ level of deconcentration requirement. With lower levels of deconcentration the MSR portfolio is able to achieve higher Sharpe ratios based on lower return but also lower level of risk relative to the RM portfolio, hence also lower indifference level of transaction costs. Finally, it should be noted that as the level of deconcentration in the efficient portfolios is increased, the indifference level of the GAMA and GAMAs portfolios changes far less than the one of RM and MSR. Furthermore, the indifference level of transaction costs increases for the RM and MSR portfolios as the deconcentration level increases until 75\%. At that level the RM portfolio experiences a fall in the indifference level of transaction costs, while for the MSR portfolio they increase further, presenting an important characteristic of the MSR portfolio regarding its out-of-sample forecasting potential. Namely, although higher deconcentration may lead to a lower in-sample Sharpe ratio, it can prove crucial in out-ofsample forecasting in order to compensate for the estimation error. Perfect diversification through high concentration in a few stocks is highly unlikely to occur out-of-sample. Also, one has to bear in mind that the main criticism of market cap-weighted portfolios is poor 
194

diversification which leads to high concentration. Even though high concentration of a capweighted index in an undeveloped market is far less pronounced in the case of CROBEX, the results of this research suggest that improvement over CROBEX could be possible.

\begin{tabular}{|c|c|c|c|c|c|}
\hline $\begin{array}{l}\text { Full sample (14 revision periods), } \lambda \\
\text { constrained }\end{array}$ & CROBEX & GAMA & GAMAs & $\mathrm{RM}$ & MSR \\
\hline Sharpe ratio (modified) & 0.30 & 0.43 & 0.43 & 0.44 & 0.48 \\
\hline Effective number of stocks & 12 & 10 & 9 & 9 & 9 \\
\hline Excess return over CROBEX index & $0.00 \%$ & $21.77 \%$ & $13.96 \%$ & $72.26 \%$ & $66.24 \%$ \\
\hline Excess turnover over CROBEX index & $0.00 \%$ & $161.68 \%$ & $146.78 \%$ & $187.76 \%$ & $193.36 \%$ \\
\hline Indifference level of transaction costs & - & $13.46 \%$ & $9.51 \%$ & $38.49 \%$ & $34.26 \%$ \\
\hline $\begin{array}{l}\text { Full sample (14 revision periods), } \\
\text { deconcentration } \approx C R O B E X \text { index } \\
\text { added }\end{array}$ & CROBEX & GAMA & GAMAs & $\mathrm{RM}$ & MSR \\
\hline Sharpe ratio (modified) & 0.30 & 0.39 & 0.40 & 0.41 & 0.43 \\
\hline Effective number of stocks & 12 & 13 & 12 & 12 & 13 \\
\hline Excess return over CROBEX index & $0.00 \%$ & $17.42 \%$ & $11.51 \%$ & $63.85 \%$ & $54.00 \%$ \\
\hline Excess turnover over CROBEX index & $0.00 \%$ & $118.08 \%$ & $119.53 \%$ & $139.63 \%$ & $139.92 \%$ \\
\hline Indifference level of transaction costs & - & $14.75 \%$ & $9.63 \%$ & $45.73 \%$ & $38.59 \%$ \\
\hline $\begin{array}{l}\text { Full sample ( } 14 \text { revision periods), } \\
\text { deconcentration } \approx 75 \% \text { added }\end{array}$ & CROBEX & GAMA & GAMAs & $\mathrm{RM}$ & MSR \\
\hline Sharpe ratio (modified) & 0.30 & 0.33 & 0.33 & 0.34 & 0.36 \\
\hline Effective number of stocks & 12 & 18 & 18 & 18 & 18 \\
\hline Excess return over CROBEX index & $0.00 \%$ & $7.91 \%$ & $5.13 \%$ & $10.76 \%$ & $37.13 \%$ \\
\hline Excess turnover over CROBEX index & $0.00 \%$ & $66.63 \%$ & $71.34 \%$ & $71.72 \%$ & $86.76 \%$ \\
\hline Indifference level of transaction costs & - & $11.87 \%$ & $7.19 \%$ & $15.00 \%$ & $42.79 \%$ \\
\hline $\begin{array}{l}\text { High volatility sample (9 revisions), } \\
\text { deconcentration } \approx 75 \% \text { added }\end{array}$ & CROBEX & GAMA & GAMAs & $\mathrm{RM}$ & MSR \\
\hline Sharpe ratio (modified) & 0.3146 & 0.3202 & 0.3214 & 0.3169 & 0.3390 \\
\hline Effective number of stocks & 12 & 18 & 18 & 18 & 18 \\
\hline Excess return over CROBEX index & $0.00 \%$ & $8.74 \%$ & $5.92 \%$ & $10.07 \%$ & $54.27 \%$ \\
\hline Excess turnover over CROBEX index & $0.00 \%$ & $74.83 \%$ & $74.14 \%$ & $74.83 \%$ & $96.60 \%$ \\
\hline Indifference level of transaction costs & - & $11.68 \%$ & $7.99 \%$ & $13.46 \%$ & $56.18 \%$ \\
\hline $\begin{array}{l}\text { Low volatility sample ( } 5 \text { revisions), } \\
\text { deconcentration } \approx 75 \% \text { added }\end{array}$ & CROBEX & GAMA & GAMAs & $\mathrm{RM}$ & MSR \\
\hline Sharpe ratio (modified) & 0.28 & 0.35 & 0.34 & 0.3861 & 0.3903 \\
\hline Effective number of stocks & 14 & 18 & 18 & 18 & 18 \\
\hline Excess return over CROBEX index & $0.00 \%$ & $6.50 \%$ & $3.79 \%$ & $11.92 \%$ & $11.60 \%$ \\
\hline Excess turnover over CROBEX index & $0.00 \%$ & $53.52 \%$ & $66.85 \%$ & $66.75 \%$ & $71.03 \%$ \\
\hline Indifference level of transaction costs & - & $12.15 \%$ & $5.67 \%$ & $17.86 \%$ & $16.33 \%$ \\
\hline
\end{tabular}

Table 4: Performance of efficient portfolios compared to the CROBEX index constrained optimisation (source: authors) 


\section{Conclusion}

In this paper we perform a comprehensive analysis of the CROBEX index in-sample efficiency, with the aim of providing information for future research efforts considering out-of-sample estimation of efficient investment strategies in the illiquid and undeveloped markets.

We find the CROBEX index to be just as inefficient as its developed market counterparts, as suggested in earlier research for the Croatian equity market by Zoričić et al. [23] and Habibović et al. [12]. However, unlike for the developed markets, the results of this research suggest that the equally-weighted index does not outperform the cap-weighted counterpart. Also, the GMV portfolio may underperform even without the transaction costs being considered. On the other hand, with the exclusion of the RR portfolio, the attractiveness of which is solely limited to risk reduction, we find that other analysed efficient portfolios do outperform the CROBEX index. Moreover, we find that they do so at a reasonably high indifference level of transaction costs, implying that an investor would not likely incur transaction costs which would offset the increase in efficiency relative to the CROBEX benchmark.

However, the analysis is conducted ex-post which implies perfect foresight. Therefore, the most valuable findings are represented by the analysis of the efficient portfolios with the level of deconcentration set to be equal or greater than the one of the CROBEX index. Namely, in an out-of-sample forecasting scenario, an investor seeking to benefit from efficient allocation has to rely on portfolio deconcentration in order to achieve diversification (extreme in-sample positions are not realistic and can be plagued with estimation error). Research findings related to this issue show that the outperformance margin of efficient portfolios is significantly reduced, especially if volatility is high. Therefore, regardless of the overall MSR portfolio dominance, research results obtained for the deconcentration level set to $75 \%$ point out the importance of considering that the estimation risk involved in the pursuit of that strategy is the highest, as estimation of 3 parameters (expected returns, covariances and variances of portfolio constituents) is required. As demonstrated by Dolinar et al. [8], significant improvement in parameter estimation is needed in order to attempt successful MSR portfolio estimation in the Croatian stock market. On the other hand, research findings in this paper suggest that RM, GAMA's and GAMA portfolios should each be considered as a viable alternative to the MSR portfolio given the relatively high indifference level of transaction costs and relatively low reduction in Sharpe ratios at the deconcentration level of $75 \%$. However, application of the Luenberger's distance function for an out-of-sample estimation of the mentioned portfolios would imply involving the same estimation risk $^{12}$ as can be seen from (2). Therefore, ultimately the research findings suggest that proxies (involving less estimation risk) for an out-of-sample estimation of the MSR portfolio should be used targeting the charachteristics of the RM, GAMA's and GAMA portfolios. Unfortunately, unlike for the developed markets as stated in Amenc et al. [2] and Amenc et al. [3], such proxies for the Croatian stock market do not include the EW and GMV portfolios as viable strategies according to the research results reported in

${ }^{12}$ Still the same 3 parameters need to be estimated. 
this paper. This implies that further research should be carried out in order to test other proposed efficient strategies for this purpose, as described in Amenc et al. [4] or their combinations as in Amenc et al. [3].

Limitations of this study are related to small data samples and observation of only 2 moments of the return distribution. Small data samples had to be used since data on daily returns are unavailable due to the illiquid market, while higher moments of return distribution could be included in the future in order to test the effect of additional risk measures on the efficient frontier and analysed efficient portfolios.

\section{References}

[1] Amenc, N., Goltz, F. and Le Sourd, V. (2006). Assessing the Quality of Stock Market Indices: Requirements for Asset Allocation and Performance Measurement, Nice: An EDHEC Risk \& Asset Management Research Centre Publication.

[2] Amenc, N., Goltz, F. and Lodh, A. (2012). Choose Your Betas: Benchmarking Alternative Equity Index Strategies. The Journal of Portfolio Management, 39(1), 88-112.

[3] Amenc, N., Goltz, F., Lodh, A. and Martellini, L. (2012). Diversifying the Diversifiers and Tracking the Tracking Error: Outperforming Cap-Weighted Indices with Limited Risk of Underperformance. The Journal of Portfolio Management, 38(3), 72-89.

[4] Amenc, N., Goltz, F. and Martellini, L. (2013). Smart Beta 2.0. Nice: An EDHEC-Risk Institute Publication.

[5] Amenc, N., Goltz, F., Martellini, L. and Retkowsky, P. (2011). Efficient Indexation: An Alternative to Cap-Weighted Indices. The Journal of Investment Management, 9(4), 1-23.

[6] Briec, W., Kerstens, K. and Lesourd, J.-B. (2004). Single period Markowitz portfolio selection, performance gauging and duality: a variation on the Luenberger shortage function. Journal of Optimization Theory and Applications, 120(1), 1-27.

[7] Chen, R. (2009). Regime Switching in Volatilities and Correlation between Stock and Bond markets. Financial Markets Group, London School of Economics and Political Science, London, UK, Discussion paper, 640.

[8] Dolinar, D., Zoričić, D. and Kožul, A. (2017). Towards the Estimation of an Efficient Benchmark Portfolio: The Case of Croatian Emerging Market. Zagreb international review of economics \& business, Special Conference Issue, April, 13-24.

[9] Gray, S. F. (1996). Modeling the Conditional Distribution of Interest Rates as a RegimeSwitching Process. Journal of Financial Economics, 42, 27-62.

[10] Grinold, R. C. (1992). Are Benchmark Portfolios Efficient? The Journal of Portfolio Management, 19(1), 34-40.

[11] Haas, M., Mittnik, S. and Paollela, M. S. (2004). A New Approach to Markov-Switching GARCH Models. Journal of Financial Econometrics, 2(4), 493-530.

[12] Habibović, A., Zoričić D. and Lovretin Golubić, Z. (2017). Efficiency of CROBEX and CROBEX10 stock market indices. UTMS Journal of Economics, 8(3), 271-280.

[13] Hamilton, J. D. (1989). A New Approach to the Economic Analysis of Nonstationary Time Series and the Business Cycle. Econometrica, 57, 357-384. 
[14] Hamilton, J. D. (1990). Analysis of Time Series Subject to Changes in Regime. Journal of Econometrics, 45, 39-70.

[15] Haugen, R. A. and Baker, N. L. (1991). The Efficient Market Inefficiency of CapitalizationWeighted Stock Portfolios. The Journal of Portfolio Management, 17(3), 35-40.

[16] Israelsen, C. L. (2005). A refinement to the Sharpe ratio and information ratio. Journal of Asset management, 5(6), 423-427.

[17] Kerstens, K., Mounir, A. and Van de Woestyne, I. (2012). Benchmarking mean-variance portfolios using a shortage function: the choice of direction vector affects rankings! Journal of the Operational Society, 63(9), 1199-1212.

[18] Luenberger, D. G. (1995). Microeconomic theory. New York: McGraw Hill.

[19] Markowitz, H. (1952). Portfolio Selection. Journal of Finance, 7(1), 77-91.

[20] Markowitz, H. (1959). Portfolio Selection: Efficient Diversification of Investments. New York: John Wiley \& Sons.

[21] Škrinjarić, T. and Šego, B. (2016). Asset allocation and regime switching on Croatian financial market. Croatian Operational Research Review, 7(2), 201-215.

[22] Tobin, J., (1958). Liquidity Preference as Behavior towards Risk. Review of Economic Studies, 25(2), 65-85.

[23] Zoričić, D., Dolinar, D. and Kožul, A. (2014). The Market Index Benchmark and Adequate Compensation for Systematic Risk in an Illiquid and Undeveloped Financial Market. In Risk Management: Strategies for Economic Development and Challenges in the Financial System, Miloš Sprčić, D. (Ed.), Nova Science Publishers Inc., New York, 257-277. 